YITP-SB-07-21

BNL-NT-07/27

\title{
Color Transfer in Associated Heavy-Quarkonium Production
}

\author{
Gouranga C. Nayak ${ }^{a}$, Jian-Wei Qiu ${ }^{b, c}$ and George Sterman ${ }^{a}$ \\ ${ }^{a}$ C.N. Yang Institute for Theoretical Physics, Stony Brook University, Stony Brook, New York 11794-3840, USA \\ ${ }^{b}$ Department of Physics and Astronomy, Iowa State University, Ames, Iowa 50011-3160, USA and \\ ${ }^{c}$ Physics Department, Brookhaven National Laboratory, Upton, NY 11973, USA
}

(Dated: August 24, 2021)

\begin{abstract}
We study the production of heavy quarkonium in association with an additional heavy pair. We argue that important contributions may come from phase space regions where three heavy fermions are separated by relative velocities much lower than the speed of light, and to which standard effective field theories do not apply. In this region, infrared sensitive color exchange is specific to the presence of the unpaired (anti)quark. This effect vanishes as the motion of the additional particle becomes relativistic with respect to the pair, and is completely absent for massless quarks and gluons in the final state.
\end{abstract}

In heavy-quarkonium production, the formation of the heavy-quark pair can be perturbative. The subsequent evolution of the quark pair is conventionally treated in the language of effective theories, most notably non-relativistic QCD (NRQCD) [1]. In NRQCD, non-perturbative dynamics is organized through a factorization characterized by a joint expansion in the strong coupling, $\alpha_{s}$ and $v$, the relative velocity. This expansion is well-justified for heavy-quarkonium decays, although it still lacks a compelling proof for production processes. It is clear, however, that NRQCD cannot apply directly to reactions in which three heavy particles, two quarks and an antiquark for example, are produced close together in phase space, simply because there is no unique choice of relative velocity $v$.

Although the application of NRQCD to production processes has had many successes [2, 3], there remain well-known data anomalies, particularly in connection with the polarization observations at the Tevatron [4] and the size of the associated production cross section for $J / \psi$ with $c \bar{c}$ pairs, as seen by the BELLE and Babar collaborations [5]. Regarding the latter, the rate for $J / \psi$ associated production is unexpectedly large compared to $J / \psi$ with light hadrons. The importance of associated production within the formalism of NRQCD has recently been explored at leading order (LO) for hadroproduction in Ref. [6] and at next-to-leading order (NLO) for $\mathrm{e}^{+} \mathrm{e}^{-}$annihilation in Ref. [7]. We will see, however, that NRQCD may not be the complete story for these processes.

Associated pair production for $J / \psi$ and related quarkonium states provides access to a novel kinematic region, where the heavy quarkonium is produced close in phase space to open heavy flavor. The presence of the additional quark, itself a source of color, could influence hadronization. In addition, studying this configuration sheds light on NRQCD factorization [8] for processes without associated heavy quark pairs, and we will make contact with our previous work on that important question [9, 10]. We begin with a discussion of matching and infrared poles in dimensional regularization for NLO corrections to associated production of heavy quarks $Q$, of mass $m$, in $\mathrm{e}^{+} \mathrm{e}^{-}$annihilation.



FIG. 1: Representative lowest order diagrams for a virtual photon decaying into two heavy quark pairs, for fixed quark momentum $P_{1}$ and antiquark momentum $P_{2}$. The pair $P_{1}$ and $P_{2}$ will be associated with a bound state.

Diagrams for $\mathrm{e}^{+} \mathrm{e}^{-} \rightarrow Q \bar{Q}+Q \bar{Q}$ are shown in Fig. 1. Because we will study final states with two pairs, we will find it useful to refer to the pair that forms the bound state as the "active" pair. To be specific, we will denote the quark and antiquark momenta of the active pair as $P_{1}$ and $P_{2}$, respectively, with total and relative momenta and rest-frame relative velocity given by

$$
P_{1}=\frac{P}{2}+q, P_{2}=\frac{P}{2}-q, v^{2}=\frac{\vec{q}^{2}}{E^{* 2}}=1-\frac{4 m^{2}}{P^{2}},
$$

where $2 E^{*}$ is the total center of mass energy of the active pair, and where in the second expression for $v^{2}$ we give the invariant form. In the diagrams of Fig. 1, we refer to the additional heavy particle that is closest in phase space to the active pair as the "spectator" quark (or antiquark).

The LO NRQCD cross sections for the amplitudes of Fig. 1 are given by

$$
d \sigma_{e^{+} e^{-} \rightarrow H+X}\left(p_{H}\right)=\sum_{n} d \hat{\sigma}_{e^{+} e^{-} \rightarrow Q \bar{Q}[n]+X}\left(p_{H}\right)\left\langle\mathcal{O}_{n}^{H}\right\rangle,
$$

where $d \hat{\sigma}_{e^{+} e^{-} \rightarrow Q \bar{Q}[n]+X}\left(p_{H}\right)$ is an NRQCD coefficient function, projected onto heavy quark pair state $Q \bar{Q}[n]$. 
The corresponding NRQCD operators are of the form [1]

$$
\mathcal{O}_{n}^{H}(0)=\sum_{N} \chi^{\dagger}(0) \kappa_{n} \psi(0)|N, H\rangle\langle N, H| \psi^{\dagger}(0) \kappa_{n}^{\prime} \chi(0),
$$

with $\kappa_{n}$ and $\kappa_{n}^{\prime}$ projections for color (octet or singlet) and spin, and where we suppress gauge links [9]. At LO, NRQCD singlet configurations are present only in diagrams where the active quark and antiquark arise from different lines, as in the first graph on the right-hand side of Fig. 1. In the second graph, the active pair is purely octet, and is expected to contribute at a much lower level to the cross section.

For NRQCD factorization to be predictive, it is necessary that the coefficient function, $d \hat{\sigma}_{e^{+} e^{-} \rightarrow Q \bar{Q}[n]+X}\left(p_{H}\right)$ be infrared safe at higher orders. Not only infrared singularities, but also singular dependence in any of the relative velocities must be absorbed into the long-distance matrix elements of the effective theory, a process referred to as matching. At LO, all gluons are off-shell by at least a multiple of $m$, and the full LO amplitudes may be absorbed into coefficient functions. Nontrivial issues of factorization and matching first arise at NLO.

It is a major undertaking to compute the full NLO corrections to the singlet NRQCD cross section [7], and all the more so for the full four-particle cross section at finite relative velocities. Nevertheless, it is relatively straightforward to check the self-consistency of the NRQCD factorization at NLO by verifying the cancellation of infrared poles.

As noted above, when more than two relative velocities are small, the standard NRQCD power counting does not apply directly, simply because there are now three dimensionless scales, $\beta_{i j} \equiv \sqrt{1-4 m^{2} /\left(P_{i}+P_{j}\right)^{2}}, i \neq$ $j=1,2,3$, instead of the single $v=\beta_{12}$ defined by the active pair. Thus, where there was once one "soft" region $m v$ and one "ultrasoft" region $m v^{2}$, there are now three of each. Let us see how these considerations emerge in the NLO diagrams of Fig. 2

We stress that the region of small relative velocity for three of the four particles in double heavy-pair production is leading-power in the overall center-of-mass energy, $\sqrt{s}$. For example, the two diagrams on the right-hand side of Fig. 1 contribute to the fragmentation of a heavy quark into the quark plus a pair. Such contributions also occur as part of the high- $p_{T}$ cross section for associated production in hadronic collisions, as studied in Ref. [6]
It is worth noting as well in this context that in such fragmentation-like contributions, the natural scale for $\alpha_{s}$ is $m$, while for the corresponding LO contributions in which a light pair recoils from a pair of heavy quarks, the natural scale for $\alpha_{s}$ is $\sqrt{s}$. This is an additional source of relative enhancement for associated production, compared to $J / \psi$ cross sections with light quanta only.

As discussed extensively in [9], factorization requires the cancellation of all infrared gluons that are not "topologically factorized". Only such contributions will match the single-pair matrix elements of NRQCD. We may think of a topologically factorized singularity as one that can either be absorbed into a quarkonium matrix element or that does not involve either of the active heavy quarks. An example of the former is the first diagram in Fig. 2, and of the latter, the second. After a sum over real gluon emission at fixed color for the active quark pair, exchanges between spectators cancel.

The third and fourth diagrams of Fig. 2, however, are not topologically factorized, and include the transfer of color between the active pair produced at short distances and the spectator. A pair produced as an octet in the hard scattering may end up as a singlet in the final state. Let us see how this mechanism enters the NLO corrections, and why it is not a problem except in the region where the spectator comes close to the active pair.

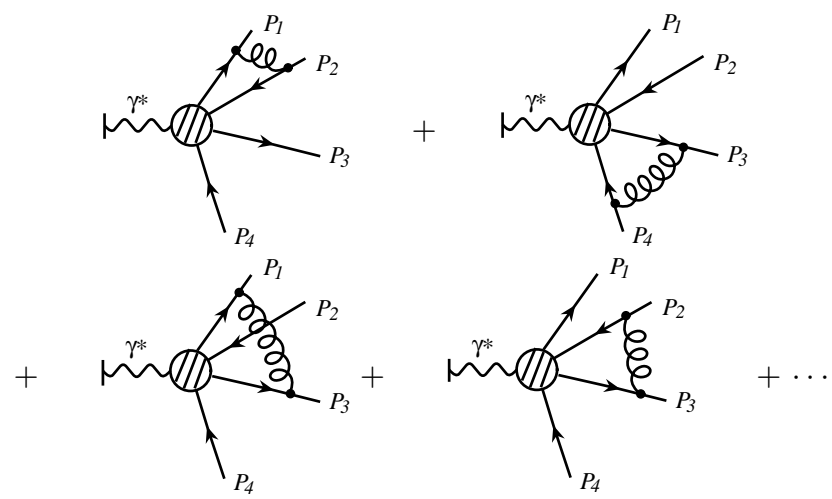

FIG. 2: Diagrams for one-loop virtual infrared corrections, where the blob represents a complete set of lowest order Feynman diagrams, as shown in Fig. 1.

When $\beta_{i j} \ll 1$, the infrared pole behavior of any of the exchanges shown in Fig. 2 is given up to a sign by

$$
-i g^{2} \int \frac{d^{D} k}{(2 \pi)^{D}} \frac{4 P_{i} \cdot P_{j}}{\left[2 P_{i} \cdot k+k^{2}+i \epsilon\right]\left[-2 P_{j} \cdot k+k^{2}+i \epsilon\right]\left[k^{2}+i \epsilon\right]}=\frac{\alpha_{s}}{2 \pi}\left[-\frac{1}{2 \varepsilon}\left(\frac{1}{\beta_{i j}}+\beta_{i j}\right)\left(2 \beta_{i j}-i \pi\right)+\ldots\right]
$$


imaginary term, which is singular for both vanishing $\beta$ and $\varepsilon$. Consider now the imaginary contributions of Eq. (4) to the NLO ampliude $\mathcal{A}$, traced over the colors of the active pair to enforce a singlet configuration in the final state. Denoting by $\mathcal{A}_{i j}$ the amplitude with an exchange between $P_{i}$ and $P_{j}$, we have, suppressing common color factors in the quark and antiquark couplings,

$$
\operatorname{Im}\left[\mathcal{A}_{13}+\mathcal{A}_{23}\right]=\frac{\alpha_{s}}{4 \varepsilon} \mathcal{A}^{(0)}\left(P_{i}\right)\left[\frac{1+\beta_{13}^{2}}{\beta_{13}}-\frac{1+\beta_{23}^{2}}{\beta_{23}}\right],
$$

where $\mathcal{A}^{(0)}$ represents the LO amplitude. From this soft gluon correction, the third and fourth diagrams in Fig. 2 inherit color singlet configurations for the pair $P_{1}, P_{2}$ in the final state at NLO. We should mention that this contribution is subleading in the number of colors $N_{c}$. Indeed, it constitutes an alternative mechanism to the "preconfinement" scenario, identified long ago in Ref. [11], and built into most hadronization models in event generators [12].

The essential feature of Eq (5) is the presence of poles in the relative velocities $\beta_{13}$ and $\beta_{23}$. These poles cancel identically for $P_{1} \cdot P_{3}=P_{2} \cdot P_{3}$ or for $P_{3}^{2}=0$. In the former case, the relative velocity of the spectator quark and the active quark equals the relative velocity of the spectator and the active antiquark. Here, soft gluons emitted by the spectator cannot resolve the charges of a perfectly co-moving pair in a singlet color state. Correspondingly, when the spectator is lightlike, its relative velocity to either active line is unity, and the two terms cancel. Thus for pair production in the presence of massless quanta only (light quark pairs and gluons) the color transfer mechanism is completely absent. This is an essential ingredient in the verification of NRQCD factorization at NNLO in Refs. [9, 10]. Evidently, a light-like spectator can neither resolve nor produce a color singlet even when the quark and antiquark are not co-moving. We see here, however, that such a cancellation requires that any heavy spectator be moving relativistically with respect to the active pair. For nonrelativistic motion, there is a nontrivial color transfer from the triplet spectator quark, that catalyzes the transition between octet and singlet.

If, as in Ref. 7], the active quark relative velocity $v$ appears only as a regulator for active-active gluon exchange, nonfactorizing singularities never occur. At the same time, at NLO, this cancellation is not exact in the low-velocity region for the spectator. The color transfer contribution is neither topologically factorized nor infrared finite, and cannot be absorbed into the coefficient function for the singlet production amplitude. Although this effect is purely imaginary at NLO, it will have a physical role at higher orders.

Since the infrared correction to the amplitude, $\mathcal{A}$ of (5) vanishes at vanishing active-pair relative momentum, it is natural to expand $\mathcal{A}$ in $v$ at fixed relative pair-spectator velocity, which we will refer to as $\beta_{S}$, between the spec- tator quark (of momentum $P_{3}$ ) and $P / 2$ [13],

$$
\beta_{S}=\sqrt{\frac{-q_{S}^{2}}{m^{2}-q_{S}^{2}}}, \quad P_{3}^{\mu}=\frac{P_{0}^{\mu}}{2} \sqrt{1-\frac{q_{S}^{2}}{m^{2}}}+q_{S}^{\mu},
$$

with $P_{0}^{\mu}=(2 m, 0)$ and $q_{S} \cdot P_{0}=0$. Expanding in the relative momentum of the active pair in the spirit of NRQCD, we find

$$
\frac{1}{\beta_{13}}-\frac{1}{\beta_{23}} \sim-\frac{4}{\beta_{S}^{3}} \frac{q_{S} \cdot q}{m^{2}} \sim \frac{4}{\beta_{S}^{2}} v \cos \phi_{S},
$$

where in the second relation $\phi_{S}$ is the angle between the vectors $\vec{q}_{S}$ and $\vec{q}$. The presence of the cosine, which is odd under the interchange of the members of the active pair, is an expression of the dipole nature of this contribution to the amplitude.

Although the expansion in Eq. (7) is applicable in only a limited region of phase space, it is a region in which the active pair and spectator quark form a system of invariant mass of order $(3 m)^{2}$. In this kinematic region, the squares of NLO corrections to LO diagrams like those on the right-hand side of Fig. 1 contribute to the cross section at leading power in the overall center-of-mass energy. At NNLO, the squares of the imaginary poles of Eq. (4) produce $\alpha_{s}^{4}\left(1 / \varepsilon^{2}\right)$ corrections to the cross section. All other diagrams contribute only at the single-pole level [13]. We note again, that in accordance with the results of Ref. 9], this contribution vanishes in the limit $m \rightarrow 0$, and so is consistent with NRQCD factorization at large $p_{T}$.

In summary, we have identified a new, leading-power "color-transfer" infrared-divergent contribution to the formation of color-singlet pairs, which is specific to associated production. This contribution must be added to that of the familiar color-octet mechanism [1]. The infrared divergence signals that color transfer cannot be absorbed into a coefficient function, while its dependence on $\beta_{S}$ signals that it cannot be absorbed into matrix elements in an effective field theory based on the dynamics of a single pair. This mechanism is non-leading in the number of colors, although that by itself should not eliminate it from consideration.

It is natural to try and estimate the magnitude of this new effect. A plausible estimate can be given by elaborating on the analogy just observed between the normal color octet mechanism and color transfer. In both cases, an $\mathrm{S}$-wave pair is transformed to $\mathrm{P}$-wave by the gluonic field strength contracted with the pair's color dipole. In the octet mechanism, the field couples to the polarization of an emitted gluon, while in color transfer it is coupled to the Coulomb field of the spectator. To return to an Swave state, we will assume a $\beta_{S}$-independent $v^{2}$ suppression, following NRQCD power counting [1, 2]. At fixed $\beta_{S}$, let us assume that the nonperturbative formation of quarkonium state, $H$ is similar for these two transitions. We then square the $\beta_{S}$-dependent kinematic factor of Eq. (77), and replace the $v$-dependence by the NRQCD matrix element $\left\langle{ }^{3} \mathcal{S}_{8}^{H}\right\rangle$. In the total cross section at fixed $\beta_{S}$, such 
a quantity would be additive to the color singlet contribution, which dominates in the NRQCD treatment. We thus have, schematically,

$$
d \sigma_{e^{+} e^{-} \rightarrow H+X}^{\text {tot }}\left(p_{H}\right) \sim d \hat{\sigma}_{e^{+} e^{-} \rightarrow Q \bar{Q}\left[S_{1}\right]+Q^{\prime}\left(\beta_{S}\right)}\left(p_{H}\right)\left\langle{ }^{3} \mathcal{S}_{1}^{H}\right\rangle+d \hat{\sigma}_{e^{+} e^{-} \rightarrow Q \bar{Q}\left[S_{8}\right]+Q^{\prime}\left(\beta_{S}\right)}\left(p_{H}\right) \frac{\left\langle{ }^{3} \mathcal{S}_{8}^{H}\right\rangle}{\beta_{S}^{4}}
$$

that is, the standard color singlet, plus a color-octet contribution enhanced by the factor $1 / \beta_{S}^{4}$, with $\beta_{S}$ the velocity of the closest heavy spectator, $Q^{\prime}=Q, \bar{Q}$. By numerical evaluation, we have verified [13] that the shortdistance cross section, $d \hat{\sigma}_{e^{+} e^{-} \rightarrow Q \bar{Q}\left[S_{8}\right]+Q^{\prime}\left(\beta_{S}\right)}\left(p_{H}\right)$ is also larger than the corresponding singlet contribution. At $\beta_{S} \sim 0.3$, our estimate of color transfer would then become competitive with the color singlet cross section at the same $\beta_{S}$. Of course, for $\beta_{S}<v$ an expansion in $v / \beta_{S}$ loses meaning, and we do not offer Eq. (8) as a realistic estimate, especially for $J / \psi$, with $v \sim 0.5$. We do present it, however, as an indication of the possible importance of the new mechanism.

A signature of the color-transfer process would clearly be an enhancement in any open heavy flavor at low relative velocity to quarkonia, in hadronic scattering as well as leptonic annihilation. Another sign could be in studies of polarization. As observed in Ref. [6] in the context of LO NRQCD, associated production leads to a more complex polarization structure than that expected from a pure octet mechanism. Although color transfer does not fall cleanly into the analysis of NRQCD, we might expect much the same spin symmetry to apply, so that a study of polarization effects can also help characterize this mechanism.

To develop an effective theory that incorporates color transfer, we shall have to add new operators. As already found in Ref. [9] in the context of NRQCD factor- ization, the long-distance transition from octet to color singlet states can be associated with a nonlocal operator of the form $\int_{0}^{\infty} d \lambda^{\prime} \lambda^{\prime}\left[P^{\mu} q^{\nu} F_{\nu \mu, a}\left(\lambda^{\prime} P\right)\right]$, with $P$ and $q$ as in Eq. (11). Noting that $\lambda^{\prime} q$ can be interpreted as the configuration-space separation of a pair, we see that this is the same operator that represents the singlet-octet transition in the potential NRQCD Hamiltonian [14, 15], here extended to production. It is clear, however, that this correspondence holds only in the region where we can strongly order the velocities. More generally, we shall have to introduce products of three fields, as $\psi_{P_{1}}^{\dagger} \chi_{P_{2}} \psi_{P_{3}}^{\dagger}$, whose interactions with the soft gluon field will be labeled by momenta (as in NRQCD) according to heavy quark effective theory. In general the nonperturbative dynamics these operators will overlap with those of NRQCD, as well as those of potential NRQCD. It will require some care to avoid double counting and to develop a formalism that can be matched to the phenomenology of associated production.

We thank Geoff Bodwin for many useful discussions on factorization. This work was supported in part by the National Science Foundation, grants PHY-0354776 and PHY-0354822, by the US Department of Energy under Grants No. DE-FG02-87ER-40371 and DE-AC02-98CH10886 and in part by the Argonne University of Chicago Joint Theory Institute (JTI) Grant 03921-07-137.
[1] G.T. Bodwin, E. Braaten and G.P. Lepage, Phys. Rev. D51, 1125 (1995), Erratum ibid. D55, 5853 (1997) arXiv:hep-ph/9407339.

[2] N. Brambilla et al. (Quarkonium Working Group) arXiv:hep-ph/0412158, and references therein.

[3] D. Acosta et al. [CDF Collaboration], Phys. Rev. D 71, 032001 (2005) arXiv:hep-ex/0412071; V. M. Abazov et al. [D0 Collaboration], Phys. Rev. Lett. 94, 232001 (2005) arXiv:hep-ex/0502030.

[4] A. A. Affolder et al. [CDF Collaboration], Phys. Rev. Lett. 85, 2886 (2000) arXiv:hep-ex/0004027; A. Abulencia et al. [CDF Collaboration], arXiv:0704.0638 [hep-ex].

[5] K. Abe et al. [Belle Collaboration], Phys. Rev. Lett. 89, 142001 (2002) arXiv:hep-ex/0205104;

B. Aubert et al. [BABAR Collaboration], Phys. Rev. D 72, 031101 (2005) arXiv:hep-ex/0506062.
[6] P. Artoisenet, J. P. Lansberg and F. Maltoni, arXiv:hep-ph/0703129

[7] Y. J. Zhang and K. T. Chao, Phys. Rev. Lett. 98, 092003 (2007) arXiv:hep-ph/0611086.

[8] M. Kramer, Prog. Part. Nucl. Phys. 47, 141 (2001) arXiv:hep-ph/0106120;

G.T. Bodwin, at 2nd International Conference on Flavor Physics (ICFP 2003), Seoul, Korea, 6-11 Oct 2003, arXiv:hep-ph/0312173

[9] G. C. Nayak, J. W. Qiu and G. Sterman, Phys. Lett. B 613, 45 (2005) arXiv:hep-ph/0501235; Phys. Rev. D 72, 114012 (2005) arXiv:hep-ph/0509021.

[10] G. C. Nayak, J. W. Qiu and G. Sterman, Phys. Rev. D 74, 074007 (2006) arXiv:hep-ph/0608066.

[11] D. Amati and G. Veneziano, Phys. Lett. B 83, 87 (1979).

[12] R. K. Ellis, W. J. Stirling and B. R. Webber, $Q C D$ and collider physics, Camb. Monogr. Part. Phys. Nucl. Phys. 
Cosmol. 8 (1996) 1.

[13] G. C. Nayak, J. W. Qiu and G. Sterman, in preparation.

[14] A. H. Hoang, 'Heavy quarkonium dynamics,' in At the Frontier of Particle Physics / Handbook of QCD, Volume 4, ed. M. Shifman (World Scientific, Singapore) p. 2215.
arXiv:hep-ph/0204299.

[15] N. Brambilla, A. Pineda, J. Soto and A. Vairo, Nucl. Phys. B 566, 275 (2000) arXiv:hep-ph/9907240. 